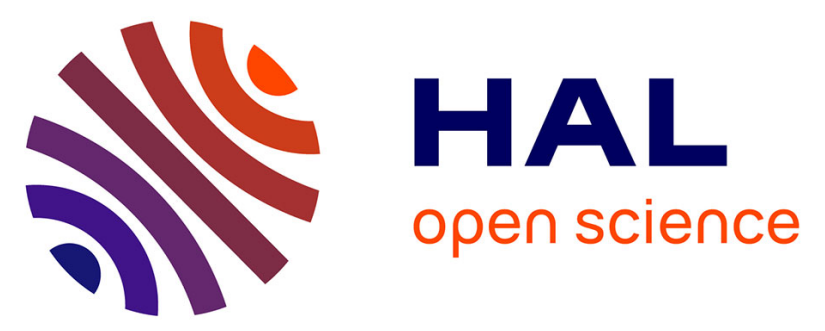

\title{
Asplünd's metric defined in the Logarithmic Image Processing (LIP) framework: A new way to perform double-sided image probing for non-linear grayscale pattern matching
}

\author{
Michel Jourlin, E. Couka, B. Abdallah, J. Corvo, J. Breugnot
}

\section{To cite this version:}

Michel Jourlin, E. Couka, B. Abdallah, J. Corvo, J. Breugnot. Asplünd's metric defined in the Logarithmic Image Processing (LIP) framework: A new way to perform double-sided image probing for non-linear grayscale pattern matching. Pattern Recognition, 2014, 47 (9), pp.2908-2924. hal00997165

\section{HAL Id: hal-00997165 \\ https://hal.science/hal-00997165}

Submitted on 28 May 2014

HAL is a multi-disciplinary open access archive for the deposit and dissemination of scientific research documents, whether they are published or not. The documents may come from teaching and research institutions in France or abroad, or from public or private research centers.
L'archive ouverte pluridisciplinaire $\mathbf{H A L}$, est destinée au dépôt et à la diffusion de documents scientifiques de niveau recherche, publiés ou non, émanant des établissements d'enseignement et de recherche français ou étrangers, des laboratoires publics ou privés. 


\title{
Asplünd's metric defined in the Logarithmic Image Processing (LIP) framework: A new way to perform double-sided image probing for non-linear grayscale pattern matching
}

\author{
M. Jourlin ${ }^{\mathrm{a}}$, E. Couka ${ }^{\mathrm{b}}$, B. Abdallah ${ }^{\mathrm{b}}$, J. Corvo $^{\mathrm{c}}$, J. Breugnot $^{\mathrm{c}, *}$ \\ ${ }^{a}$ Laboratoire Hubert Curien, UMR CNRS 5516, \\ 18, Rue du Professeur Benot Lauras, 42000 Saint-Etienne (France) \\ ${ }^{b}$ Centre de Morphologie Mathematique, \\ 35, rue Saint Honor, 77305 Fontainebleau (France) \\ ${ }^{c}$ SILAB, Digital Imaging Platform, \\ ZI de la Nau, 19240 Saint-Viance (France)
}

\begin{abstract}
The present paper focuses on non-linear pattern matching based on the Logarithmic Image Processing (LIP) Model. Our contribution consists first of using the scalar multiplication defined in the LIP context to extend the little-known Asplünd's metric to gray level images. Such a metric is explainable as a novel technique of double-sided image probing and presents the decisive advantage of being physically justified in the field of transmitted light acquisition. Moreover, thanks to the consistency of the LIP context with human vision, Asplünd's metric is also applicable to images acquired in reflected light: in fact, plenty of image processing algorithms aim at extracting information as a human eye would do. Finally, the proposed approach is particularly efficient in presence of lighting variations or lighting drift. In the paper, we also suggest a solution to overcome the main drawback of probing techniques, which resides in a high sensitivity to noise. Various examples are presented to highlight the efficiency of the method.
\end{abstract}

Keywords: Grayscale pattern matching, Probing, LIP Model, Metrics.

\section{Introduction}

Pattern matching consists in detecting occurrences of a given template within a search image. This topic has been widely investigated by researchers involved in target tracking, artificial vision, characterization of pseudo-periodic

\footnotetext{
* Corresponding author

Email addresses: michel.jourlin@univ-st-etienne.fr (M. Jourlin), enguerrand.couka@mines-paristech.fr (E. Couka), bassam.abdallah@mines-paristech.fr (B. Abdallah), joris.corvo@live.fr (J. Corvo), j.breugnot@silab.fr (J. Breugnot)
} 
textures, image analysis, registration, etc. Most of pattern matching algorithms are founded on the use of correlation tools or metrics in order to estimate the similarity between the template and a subset of the studied image, or simply between two images.

Let us refer to some other examples of metric-based pattern matching. HelOr et al. (HelOr, 1985) decrease the computation time thanks to projection kernels allowing a rapid rejection of image windows that are distant from the pattern. Bozkaya et al. (Bozkaya, 1997) aim at retrieving all images of a data base that are similar to a given query image. In the same objective to estimate similarity between a template and a region of the studied image, correlation is a very popular tool (Gruen, 1985).

Generally, the notion of similarity refers to a "good" superposition of the considered images representative surfaces. An example of such an approach is given by Huttenlocher et al. (Huttenlocher, 1993) and is based on the Hausdorff metric. A recurrent problem of such methods resides in their high sensitivity to illumination variations, pattern size changing and generally small evolutions of image acquisition conditions. In case of variable illumination conditions, D. Lefebvre et al. (Lefebvre, 2002) propose a technique of correlation peaks that are invariant under linear intensity transformation.

In (Barat, 2003) and (Barat, 2010), Barat et al. proposed an alternative way for detecting a known template within an image: the Morphological Probing (Barat, 2003), and the Virtual Double-Sided Probing (VDIP, (Barat, 2010)). The VDIP consists of defining an inferior probe and a superior probe. In their reference configuration, the probes form a template, which includes all objects to detect. These objects may be for example occurrences of a unique model with varying size or varying aspect due to noise or illumination changes. The template specifies the distortion constraints and fixes the limit of variability of a given query pattern. The present paper is an extension of this approach based on a new notion of probing: the Asplünd's metric.

The little-known Asplünd's metric was initially defined on binary shapes (Asplund, 1960), (Grünbaum, 1963) and we have extended this notion to gray tone images (Jourlin, 2012). The specificity of the pattern matching approach proposed in this paper is linked to an outstanding property of the Asplünd's metric: it is independent of possible magnifications of the studied binary shapes. In the gray tone context, this property will result in a strong independence of Asplünd's metric to lighting variations. Furthermore, Asplünd's metric computation is founded on the use of two homothetic functions of the considered template in order to obtain the upper and lower probing sets of the studied image. Such homothetic functions are here defined in the LIP Model framework (Jourlin, 1988), (Jourlin, 2001) which gives them both a precise physical meaning and a consistency with human vision (Brailean, 1991). 
In section 2 the definition of binary Asplünd's metric is recalled, as well as the necessary notions issued from the LIP Model. Section 3 is devoted to the gray tone version of Asplünd's metric with a discussion of its advantages, drawbacks and applicative efficiency. In section 5 a metric issued of "Measure Theory" is presented and used to overcome the noise sensitivity of Asplünd's metric. We conclude and present the perspectives of this work in section 6 .

\section{Preliminaries}

\subsection{About metrics and associated topologies}

In this section, the aim is not to enter in depth in the field of general topology, because our paper focuses on the particular case of metrics and precisely on the notion of Asplünd's metric. Let us begin with a brief recall on the most common notions used to estimate the similarity between two binary shapes A and $\mathrm{B}$ of the plane $R^{2}$ (if necessary of $R^{3}, . ., R^{n}$ ).

\subsubsection{Distances between binary shapes}

- Symmetrical difference metric

The "symmetrical difference" of $\mathrm{A}$ and $\mathrm{B}$ is noted $A \triangle B$ and is defined according to:

$$
A \triangle B=(A \cup B) \backslash(A \cap B)
$$

The Symmetrical Difference distance $d_{\triangle}(A, B)$ corresponds then to the area of $A \triangle B$ (cf. Fig. 1 (a)).

\section{- Hausdorff metric}

The definition of the Hausdorff distance $d_{H}(A, B)$ is given by the following formula:

$$
\begin{aligned}
d_{H}(A, B) & =\operatorname{Max}\left[\sup _{a \in A} \inf _{b \in B} d(a, b), \sup _{b \in B} \inf _{a \in A} d(a b)\right] \\
& =\operatorname{Max}\left[\sup _{a \in A} d(a, B), \sup _{b \text { inB }} d(b, A)\right]
\end{aligned}
$$

It represents the maximal Euclidean distance between an element of $A$ (or $B$ ) and $B$ (or $A$ ) (cf. Fig. 1 (b)) 


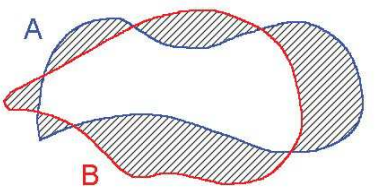

(a)

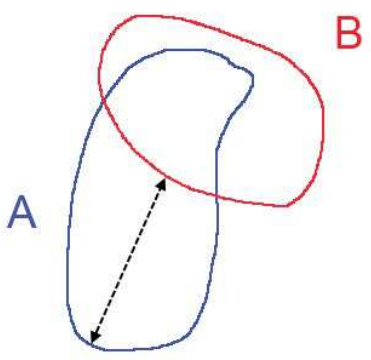

(b)

Figure 1: Illustration of what $d_{\triangle}(A, B)$ and $d_{H}(A, B)$ respectively represent: (a) The hatched area represents $d_{\triangle}(A, B)$, (b) the dotted line represents $d_{H}(A, B)$

Comments: The previous metrics do not estimate the similarity between A and B in the same way. In fact, $d_{\triangle}$ is of a "global" or "diffuse" nature in the sense that the similarity between the two shapes is roughly evaluated: small sized differences will not be detected. On the opposite, $d_{H}$ will put in evidence such small differences (even reduced to a single point) and is then said of a "local" or "atomic" nature.

\section{- Binary Asplünd's metric}

Now let us introduce a completely different approach proposed by Asplünd (Asplund, 1960), (Grünbaum, 1963). Given two shapes $A$ and $B$, one of them ( $B$ for example) is chosen as the "probing" shape: we compute the smallest homothetic set $\lambda B$ containing $A$ and the largest homothetic set $\mu B$ included in $A$ (Fig. 2). It means:

$$
\begin{aligned}
& \lambda=\inf \{\alpha, A \subset \alpha B\} \\
& \mu=\sup \{\beta, \beta B \subset A\}
\end{aligned}
$$

where $\alpha$ and $\beta$ are positive real numbers.

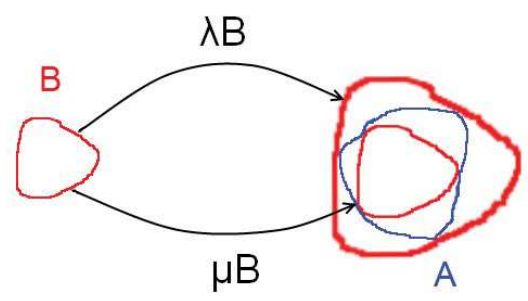

Figure 2: Probing of A by B 
The Asplünd's distance $d_{A s}(A, B)$ is given by:

$$
d_{A s}(A, B)=\operatorname{Ln}\left(\frac{\lambda}{\mu}\right)
$$

Comments: the major interest of the Asplünd's similarity evaluation between two shapes resides in its invariance when one of the shapes is magnified or reduced in an arbitrary ratio $\alpha$. In fact, replacing the probing set $B$ by $\alpha B$ does not modify the resulting Asplünd's distance:

$$
d_{A s}(A, \alpha B)=\operatorname{Ln} \frac{\frac{\lambda}{\alpha}}{\frac{\mu}{\alpha}}=\operatorname{Ln} \frac{\lambda}{\mu}=d_{A s}(A, B)
$$

In the context of gray level functions (Section 3), this property of Asplünd's metric will be interpreted as a strong stability in presence of lighting variations. Before that, some "functional" metrics are presented.

\subsubsection{Distances between gray level functions}

Let us recall the notions of distances corresponding to $d_{\triangle}$ and $d_{H}$ (respectively $d_{1}$ and $d_{\infty}$ ) between two gray level functions $f$ and $g$.

Such functions are defined on a spatial domain $D$ (subset of the plane $R^{2}$ ), with values in the gray scale [0, $M$ [ where $M$ represents the available number of gray levels: If the gray level functions are digitized onto 8 bits, $M=2^{8}=256$.

\section{- Metric $d_{1}$}

The $d_{1}$ distance associates to a pair $(f, g)$ of gray level functions defined on $D$ or a region $R$ of $D$ the number:

$$
d_{1, D \text { or } R}(f, g)=\iint_{D \text { or } R}|f(x, y)-g(x, y)| d x d y
$$

In the digital version, it is transformed into the double sum of the differences between pixels gray levels according to the rows and columns, multiplied by the area of one pixel. It thus evaluates the "volume" situated between the representative surfaces of images $f$ and $g$ :

$$
\begin{aligned}
d_{1, D \text { or } R}(f, g)= & {\left[\sum_{(i, j) \in D \text { or } R}|f(i, j)-g(i, j)|\right] } \\
& \times \text { area of a pixel }
\end{aligned}
$$

Comments: As previously announced, such a distance is comparable to $d_{\triangle}$ in the sense it produces a global evaluation of the similarity between $f$ and $g$ (Fig. 3 (a)) and it is weakly sensitive to small sized differences. 


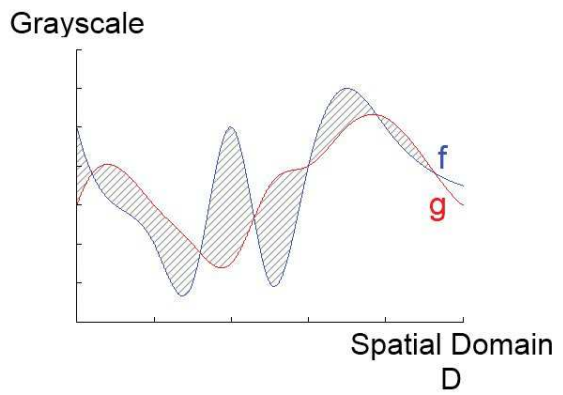

(a)

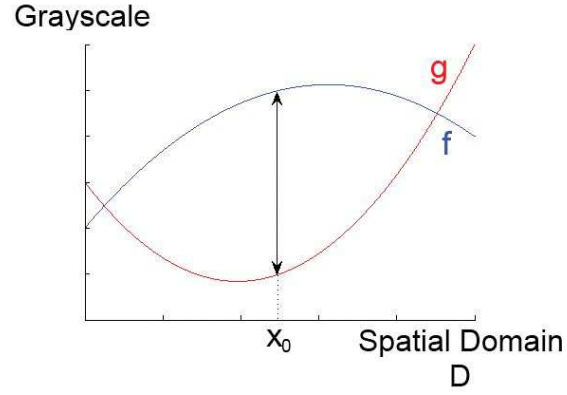

(b)

Figure 3: (a) The value of $d_{1}(f, g)$ corresponds to the hatched area between the representative surfaces (here curves) of $f$ and $g$, (b) The distance $d_{\infty}(f, g)$ is realized at the point $x_{0}$

\section{- Metric $d_{\infty}$}

On the contrary, we can use "atomic" metrics, analogue to measures using "weighted" points (Dirac measures). They are then perfectly adapted in detecting small differences, even as small as a pixel (Fig. 3-b) and are then similar to the binary Hausdorff metric $d_{H}$. The most typical example is the metric $d_{\infty}$ derived from the norm of uniform convergence in the $L^{\infty}$ space, which is computed on the point realizing the greatest difference between $f$ and $g$ :

$$
d_{\infty}(f, g)=\sup _{(x, y) \in R \text { or } D}|f(x, y)-g(x, y)|
$$

In digital version :

$$
d_{\infty}(f, g)=\sup _{(i, j) \in R \text { or } D}|f(i, j)-g(i, j)|
$$

Remark: Note that various Hausdorff metrics exist in the functional domain i.e. applicable to functions and in our case to gray level images (cf. for example (Friel, 1998), (Odone, 2001), (Girard, 2010)). Such metrics have a typical "atomic" behavior: it is the reason why we have deliberately made the choice to limit our interest to $d_{\infty}$.

\subsubsection{Neighborhoods generated by the previous metrics}

One of the major interests of the "metric" tool resides in its associated topology, i.e. in the neighborhoods it generates. The shapes of such neighborhoods are totally different for the $d_{1}$ and $d_{\infty}$ metrics. In fact, given a function $\mathrm{f}$, each function $g$ verifying $d_{\infty}(f, g) \leq \epsilon$ satisfies $|f(x)-g(x)| \leq \epsilon$ for every point $x$ lying in the considered interval or region. In one dimension, it means that $g$ belongs to a "tolerance tube" around f (Fig. 4 (a)). This remark explains why $d_{\infty}$ is called "uniform convergence metric". The same result holds for images, the tolerance tube becoming the volume located between the translated representative surfaces of $f$ according to $+\epsilon$ and $-\epsilon$. 


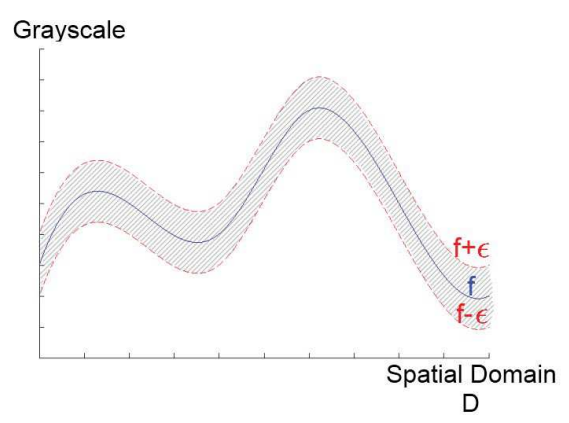

(a)

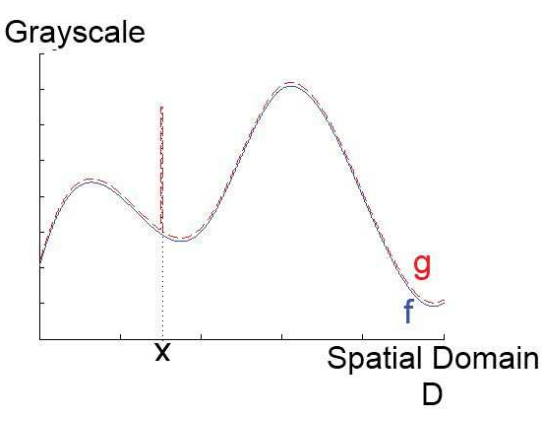

(b)

Figure 4: (a) Representation of the tolerance tube of $\mathrm{f}$ (hatched area), (b) the difference between a function $f$ and a function $g$ lying in the $\epsilon$-neighbor of $f$ may be arbitrarily large at some point $x$

When considering the "global" metric $d_{1}$, a $\epsilon$-neighbor of a given function $f$ is totally different from a tube: it is an unbounded set! In fact, a function $g$ belonging to the $\epsilon-$ neighbor of $f$ may present at some point $x$ an arbitrary large difference $|f(x)-g(x)|$ and a very small area located between $f$ and $g$ (Fig. 4 (b)).

\subsubsection{Strategy to define a new "probing" metric}

In order to create a new double-sided probing, the following steps will be considered in the next sections:

- section 2.2 : Recalls on the Logarithmic Image Processing (LIP) Model, which constitutes the adequate mathematical framework to define the multiplication of a gray level function $f$ by a real number $\lambda$, noted $\lambda \otimes x$.

- section 3: Extension of the binary Asplünd's metric to gray level functions, thanks to the previous scalar multiplication.

\subsection{Logarithmic Image Processing (LIP) Model}

Introduced by Jourlin et al (Jourlin, 1988), (Jourlin, 2001), the LIP (Logarithmic Image Processing) Model proposes first a framework adapted to images acquired in transmitted light (when the observed object is placed between the source and the sensor). In this context, each gray level image may be identified to the object, as long as the acquisition conditions (source intensity and sensor aperture) remain stable. Furthermore, the demonstration, by Brailean (Brailean, 1991) of the LIP Model compatibility with human vision, considerably enlarges the application field of the Model, particularly for images acquired in reflected light on which we aim at simulating human visual interpretation. 
An image $f$ is defined on a spatial support $D$, with values in the gray scale $[0, M[$, which may be written:

$$
f: D \subset \mathbb{R}^{2} \rightarrow[0, M[\subset \mathbb{R}
$$

In the LIP context, 0 corresponds to the "white" extremity of the gray scale, which means to the source intensity, i.e. when no obstacle (object) is placed between the source and the sensor. Thanks to this gray scale inversion, 0 will appear as the neutral element of the logarithmic addition (formula 3 below).

The other extremity $M$ is a limit situation where no element of the source is transmitted (black value). This value is excluded of the scale, and when working with 8-bits digitized images, the 256 gray levels correspond to the interval of integers $[0, \ldots, 255]$.

The transmittance $T_{f}(x)$ of an image $f$ at $x \in D$ is defined by the ratio of the outcoming flux at $x$ to the incoming flux (intensity of $S$ ).

In a mathematical formulation, $T_{f}(x)$ may be understood as the probability, for a particle of the source incident at $x$, to pass through the obstacle, which means to be seen by the sensor.

The addition of two images $\mathrm{f}$ and $\mathrm{g}$ corresponds to the superposition of the obstacles (objects) generating respectively $\mathrm{f}$ and $\mathrm{g}$. The resulting image will be noted:

$$
f \AA g
$$

Such an addition is strongly linked to the transmittance law

$$
T_{f \triangle_{g}}=T_{f} \times T_{g}
$$

It means that the probability, for a particle emitted by the source, to pass through the "sum" of the obstacles $f$ and $g$, equals the product of the probabilities to pass through $f$ and $g$, respectively.

Jourlin and Pinoli (Jourlin, 2001) established the link between the transmittance $T_{f}(x)$ and the gray level $f(x)$ :

$$
T_{f \AA_{g}}=1-\frac{f(x)}{M}
$$

Replacing in formula (1) the transmittances by their values obtained in (2) yields:

$$
f \AA g=f+g-\frac{f \cdot g}{M}
$$

From this law, it is possible to derive the multiplication of an image by a positive real number $\lambda$ according to :

$$
\lambda \otimes f=M-M\left(1-\frac{f}{M}\right)^{\lambda}
$$


Fundamental remark: such laws satisfy strong mathematical properties. In fact, if $\mathcal{I}(D,[0, M[)$ and $\mathcal{F}(D]-,\infty, M[)$ design respectively the set of images defined on $D$ with values in [0, $M[$, and the set of functions defined on $D$ with values in $]-\infty, M[$, we have:

$$
(\mathcal{F}(D,]-\infty, M[), \AA, \AA) \text { is a real vector space }
$$

$(\mathcal{I}(D,[0, M[), \triangle, \otimes)$ is the positive cone of this vector space

(for more details, see (Jourlin, 2001)).

\section{A new probing approach for template location: Asplünd's metric for gray tone images}

\subsection{Definition}

It seems us very interesting to extend Asplünd's reasoning in a "functional" context, in order to apply it to gray level images:

- a first generalization would consist of using classical homothetic functions of $f$, noted $\lambda f$ to propose Asplünd-like metrics. This approach presents a certain weakness because the homothetic $\lambda f$ of an image not always remains in the gray scale.

- the novelty of what we proposed in (Jourlin, 2012) is to replace an ordinary homothetic function $\lambda f$ by a logarithmic homothetic $\lambda \otimes f$.

Given two images $f$ and $g$ defined on $D$, we choose, as for binary shapes, $g$ as the probing function for example and define the two numbers:

$$
\lambda=\inf \{\alpha, f \leq \alpha \otimes g\} \text { and } \mu=\sup \{\beta, \beta \otimes g \leq f\}
$$

and the corresponding "functional Asplünd's metric" $d_{A s}^{\lambda}$ :

$$
d_{A s}^{\hat{\lambda}}(f, g)=\operatorname{Ln}\left(\frac{\lambda}{\mu}\right)
$$

Physical interpretation: Asplünd's metric being directly based on the scalar multiplication law defined in the LIP context, it is important to precise the physical meaning of this law. In fact, in situation of transmitted light, computing $2 \otimes f=f \AA f$ consists of stacking up two times the semi-transparent object corresponding to $\mathrm{f}$. More generally, the scalar multiplication of the image $\mathrm{f}$ by $\lambda$ is explainable as a thickness changing of the observed object in the ratio $\lambda$ : if $\lambda>1$, the thickness increases and the resulting image is darker than $f$ and if $\lambda<1$, the thickness decreases and the resulting image is brighter than $f$ (cf Fig. 5). This point is fundamental and will explain the particular efficiency of Asplünd's metric in presence of lighting variations or lighting drift, as long as such variations may be modeled by thickness changing.

\section{Remarks:}




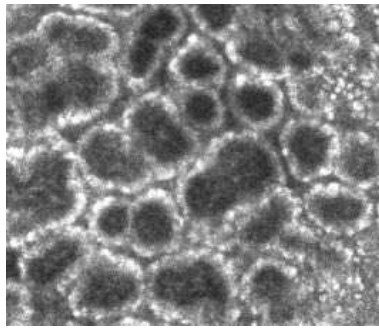

(a)

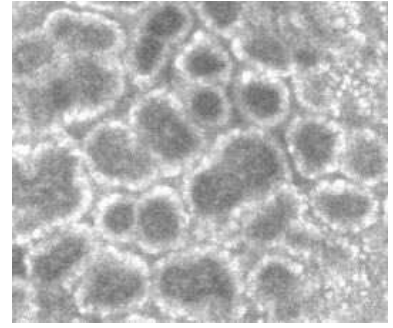

(b)

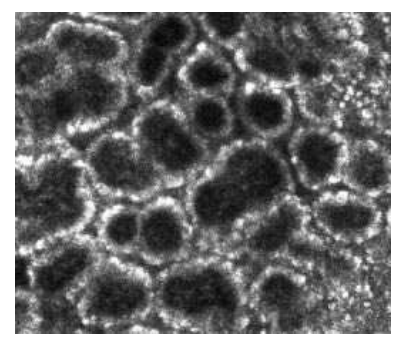

(c)

Figure 5: Brightening /darkening an image by means of the LIP scalar multiplication : (a) Initial image $f$ of dermal papillae by in-vivo confocal microscopy, (b) Image $\lambda \widehat{A} f$ for $\lambda=0.5$, (c) Image $\lambda \widehat{\triangle} f$ for $\lambda=1.5$

- To compute formula 5 in good conditions, we must take care that every gray level present in the chosen template is not null (if not, the homothetic values $\lambda$ and $\mu$ may reach infinity.

- A simple solution to avoid such a situation consists of adding one unit to each gray level of the considered image.

- This metric $d_{A s}^{\hat{X}}$ is adaptable to local processing, in particular to detect on an image $f$ the place where a given template is probably located. In this case, the template corresponds to an image $t$ defined on a spatial support $D t$ smaller than $D$. For each location of $D t$ included in $D$, the distance $d_{A s}^{\widehat{X}}\left(\left.f\right|_{D t}, t\right)$ is computed, where the notation $\left.f\right|_{D t}$ represents the restriction of $f$ to $D t$.

Then such distances are normalized to cover the gray scale $[0,255]$ and the darkest areas correspond to minimal distances. The result is visualized under the name of Asplünd's map, which constitutes the first step of pattern recognition.

\subsection{Examples}

Let us now visualize some images on which a target has been selected and the corresponding Asplünd's maps computed. Remember that Asplünd's approach holds in both situations of images acquired in transmission or reflection.

\subsubsection{Images acquired in transmission}

Petri dishes. In order to locate the bacteria present in a Petri dish (Fig. 6 (a), we select a target (Fig. 6 (b) and compute the Asplünd's map (Fig. 6.c) corresponding to this target when we move it inside the Petri dish. 


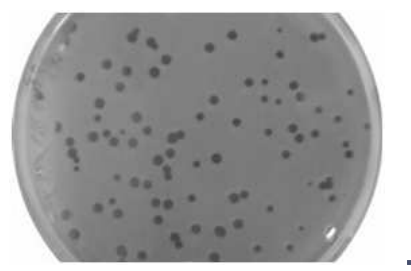

(a)

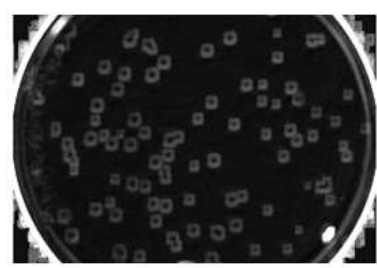

(c)

Figure 6: (a) Petri dish (Escherichia Coli bacteria), (b) Target selected inside a bacterium (dark zone), (c) Asplünd's map

Comments on Figure 6: On the Asplünd's map, we can observe the location of the bacteria is obtained. The bright zone surrounding each of them corresponds to high values of Asplünd's metric, when the target meets the background. Note also that the distance is small when the target is included in the background, due to the probing effect.

Image of human skin : dermoepidermal junction acquired in in-vivo confocal microscopy. We tackle here a problem of higher complexity than the previous one. Nevertheless, the steps are the same: target selection and creation of Asplünd's map (cf. Fig. 7).

Comments on Figure 7; The initial image Fig. 7(a) is acquired in transmission. An automated extraction of the dark regions representing the inner boundaries of the papillae is a hard problem if we consider their heterogeneity. The target Fig. 77 (b) is a subset of Fig. 7 (a) selected into a dark region. Nevertheless, this pattern is not homogeneous in terms of gray levels, as it can be seen on the magnified image Fig. 7 (c). At each position of the target inside the initial image, the local Asplünd's distance is computed and the map of such distances is represented on Fig. 7 (d). The dark points correspond to the target locations where small distances have been computed. Note that the dark regions are now rather homogeneous. Thus, to get a binary image, it remains to apply to d) an automated thresholding algorithm (Interclass Variance Maximization of (Otsu, 1979) for example. Morphological operations (opening and closing) are then applied in order to smooth the boundaries. These last one are superposed on the initial image (Fig. 7(e)).

\subsubsection{Images acquired in reflection}

Remember that (Brailean, 1991) established the consistency of LIP Model operators with human visual system. This result justifies the use of Asplünd's metric to process images acquired in reflection, as long as the information we aim at, corresponds to human interpretation.

Target detection in images of car crash test. The Asplünd's distance is used here to detect the targets during a car crash test (Fig. 8 (a) with authorization of the Insurance Institute for Highway Safety). As seen above, we need a probing 


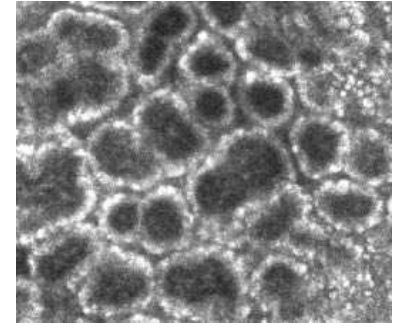

(a)

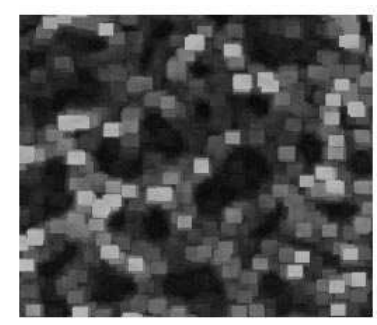

(d)

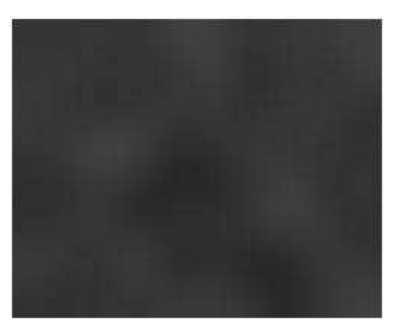

(c)

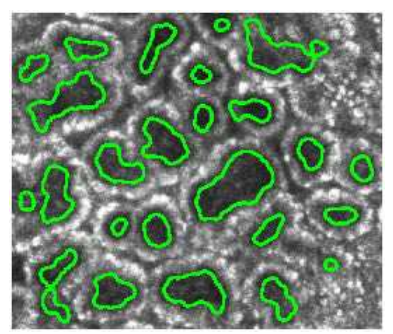

(e)

Figure 7: Asplünd's detection of dermal papillae : (a)Initial image of dermal papillae by invivo confocal microscopy, (b) Selected target, (c) Magnified target, (d) Asplünd's maps, (e) Contours extracted from (d) and superposed on (a)

function which will fit into the target we are looking for. On Fig. 8 (b), the white area delimitates the function (target subset) which will probe the image. In fact, the different locations of the target inside the initial image may be not exactly the same by their orientation, their size, etc. The probing function is chosen smaller than the target in order to adapt to these small variations. On Fig. 8 (c), the darkest areas correspond to a small distance, and a bright area to a high distance. All the targets have been detected, except the one on the head of the dummy. This is due to the different orientation of this target (more than $45^{\circ}$ with the reference).

Pores detection on human skin. The initial image (Fig 9 a) represents human skin. The location of pores inside this image is rather difficult, due to the variations of shapes and gray levels of such pores. We selected a pore on the initial image and represented it (Fig. 9-b) magnified twenty times. Then we obtain the map of Asplünd's distances (Fig. 9-c) when moving the template inside the initial image. To conclude, an automated thresholding (Variance Interclass Maximization, cf. (Otsu, 1979)) applied to this map produces the pores locations (Fig. 9fd).

\subsection{Neighborhoods associated to Asplünd's metric}

\subsubsection{Tolerance tubes}

First let us recall the above mentioned "comment" (following Fig. 2), pointing the invariance of Asplünd's metric when one of the shapes is magnified or 


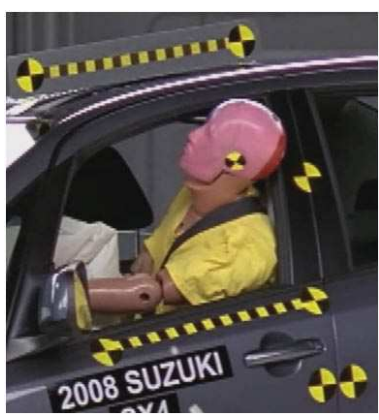

(a)

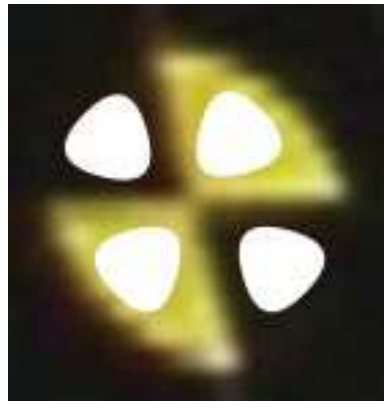

(b)

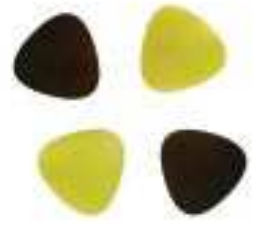

(c)

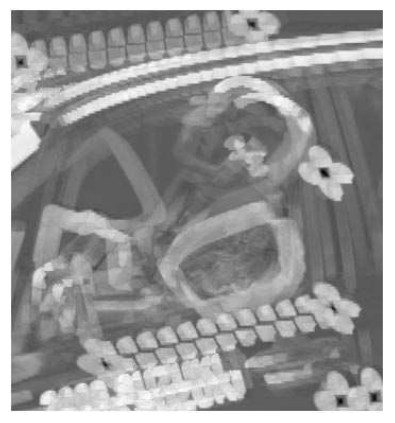

(d)

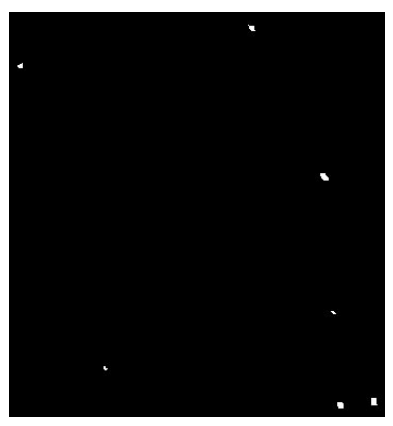

(e)

Figure 8: Target detection thanks to Asplünd's metric : (a) Crash test Image, (b) Probing zones inside the target magnified 10 times, (c) Probing function magnified 10 times, (d) Asplünd's map, (e) Thresholded image of (d) : location of the targets 


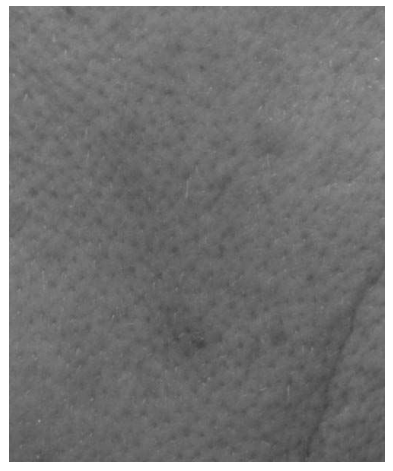

(a)

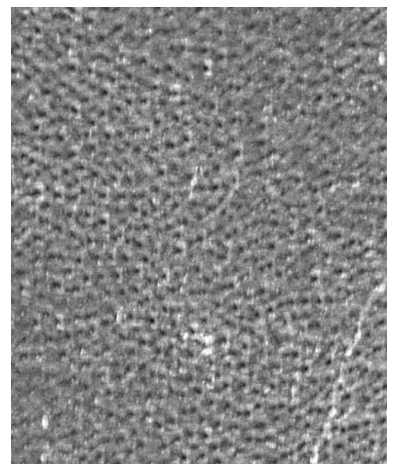

(c)

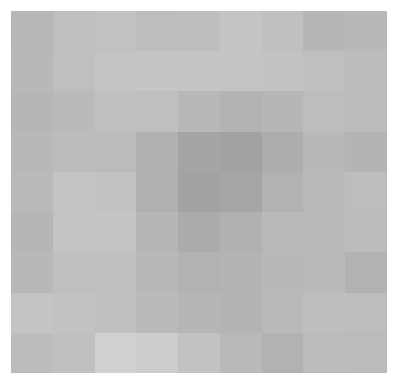

(b)

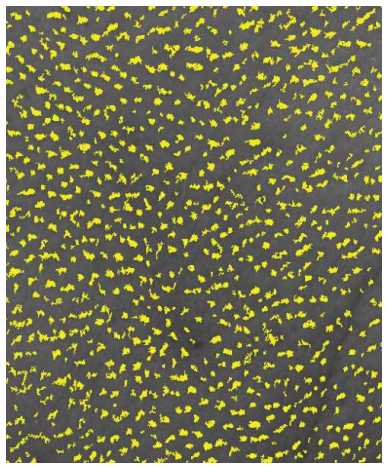

(d)

Figure 9: Occurrences of a given template on a human skin image : (a) Initial image: human skin with selected template (white boundary), (b) Chosen template (a skin pore) magnified 20 times), (c) Map of Asplünd's distances when moving the template inside the image, (d) Pores detection on image (a) (automated thresholding on (c)) 


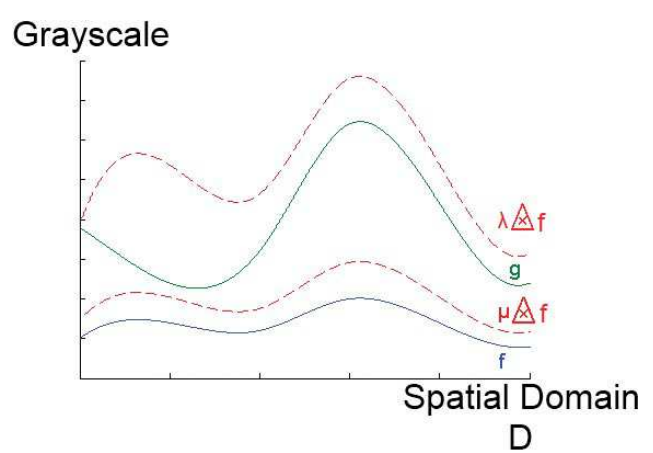

Figure 10: A tolerance tube $T_{\lambda, \mu, \epsilon}(f)$ delimited by $\lambda \otimes f$ and $\mu \widehat{x} f$ and a neighbor $g$ of $f$ lying in this tube

reduced in an arbitrary ratio $\alpha$.

The same property holds for gray level functions:

If $d_{A s}^{\widehat{\lambda}}(f, g)=\operatorname{Ln}\left(\frac{\lambda}{\mu}\right)$, we can write $d_{A s}^{\widehat{\lambda}}(f, \alpha \otimes g)=\operatorname{Ln} \frac{\frac{\lambda}{\frac{\alpha}{\alpha}}}{\alpha}=d_{A s}^{\otimes}(f, g)$.

Given an image $f$ and a tolerance $\epsilon$, we can consider the following successive steps:

- Create a family of tolerance tubes $T_{\lambda, \mu, \epsilon}(f)$ constituted of regions delimited by two homothetics $\lambda \otimes f$ and $\mu \widehat{x} f$ such that $\operatorname{Ln}\left(\frac{\lambda}{\mu}\right)=\epsilon$.

- Define the neighborhood $N_{A s, \epsilon}^{\widehat{x}}(f)$ :

$$
\begin{gathered}
g \in N_{A s, \epsilon}^{\widehat{\lambda}}(f) \Leftrightarrow \exists(\lambda, \mu), \exists \alpha, / \operatorname{Ln}\left(\frac{\lambda}{\mu}\right)=\epsilon \\
\text { and } \alpha \widehat{\otimes} g \in T_{\lambda, \mu, \epsilon}(f)
\end{gathered}
$$

- Visualize a mono-dimensional representation (Fig. 10), which perfectly illustrates the Asplünd's property underlined in the comments at the end of 2.1.1. One image or a template $g$ may be a neighbor of an image $f$, in Asplünd's sense, even if $g$ is significantly darker (or brighter) than $f$ : it suffices that some homothetic of $g$ resembles some (other) homothetic of $f$.

This point must be considered as an advantage if the template and the search image are for example acquired under different illumination conditions. On the opposite, it could be a real problem if the resemblance between the template 


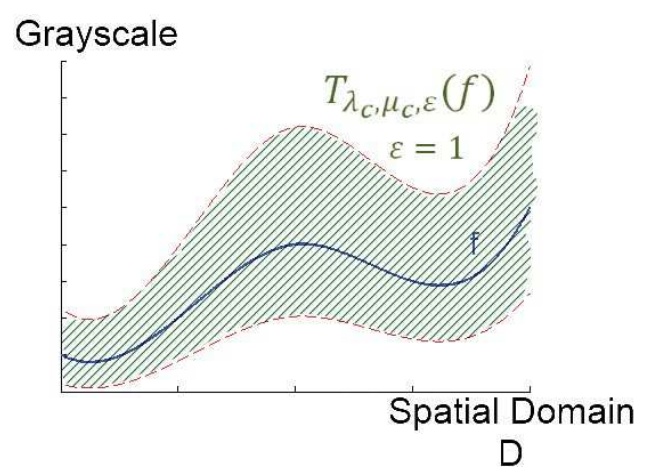

Figure 11: A given function $\mathrm{f}$ and the centered tube $T_{\lambda c, c, \epsilon}(f)$

and the image is intended in terms of gray levels. For this reason, let us show that a "centered" tolerance tube may be selected within the family of all the tolerance tubes.

\subsubsection{Tolerance tube centered on the studied gray level function}

Given a gray level image $f$, and a tolerance value $\epsilon$ in terms of Asplünd's metric, the neighbor $N_{A s, \epsilon}^{\mathrm{X}}(f)$ is the union of all the tubes $T_{\lambda, \epsilon}(f)$. Among them, consider the tube $T_{\lambda c, \mu c, \epsilon}(f)$ defined by a pair of real numbers $\lambda c$ and $\mu c$ compelled to have symmetric values around the unit integer 1 , for example such that $\lambda c=1+k$ and $\mu c=1-k$. Such a tube is centered at $f$ and the condition $\operatorname{Ln}\left(\frac{\lambda c}{\mu c}\right)=\epsilon$ yields:

$$
\begin{aligned}
\operatorname{Ln}\left(\frac{1+k}{1-k}\right)=\epsilon & \Leftrightarrow \frac{1+k}{1-k}=e^{\epsilon} \\
& \Leftrightarrow k=\frac{e^{\epsilon}-1}{e^{\epsilon}+1}
\end{aligned}
$$

which gives the values of $\lambda c$ and $\mu c$ :

$$
\lambda c=\frac{2 e^{\epsilon}}{e^{\epsilon}+1} \text { and } \mu c=\frac{2}{e^{\epsilon}+1}
$$

The resulting tube is represented in Fig. 11. On the brick wall image of Fig. 15 (a), it is then possible to extract only the bricks presenting gray levels resembling those of a given template $T$ (cf Fig. 12) 


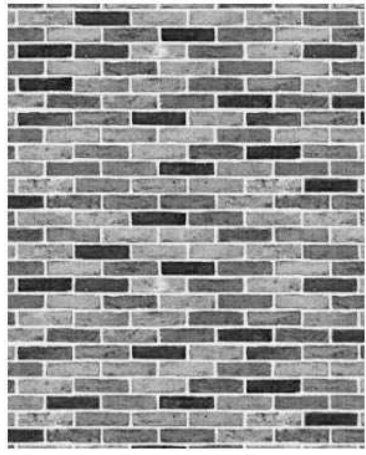

(a)

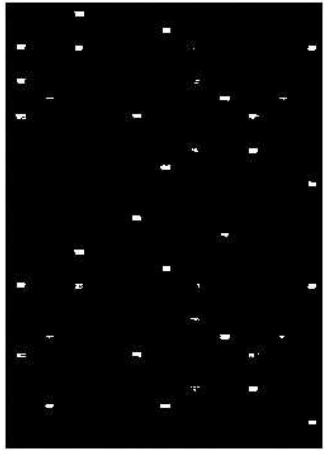

(c)

Figure 12: Application of a centered tube : (a) Initial image, (b) template $T$, (c) Locations, after thresholding of the Asplünd's map, of bricks (the dark ones) belonging to the tube $T_{\lambda c, \mu c, \epsilon}(T)$ for $\epsilon=1$

\section{Comparison of Asplünd's metric with other Pattern Recognition techniques}

\subsection{Comparison with other metrics}

We will adopt here the definitions and notations of section 2.1.2 devoted to classical distances between gray level functions.

\subsubsection{Global metrics}

First let us note that "global" distances like $d_{1}, d_{2}, \ldots, d_{p}$ considerably differ from Asplünd's approach:

- They are unable to detect small sized objects

- They do not generate tolerance tubes (cf. section 2.1 devoted to neighborhoods associated to the considered metrics)

In conclusion, such metrics are not really comparable to Asplünd's one and do not aim at the same applications.

\subsubsection{Atomic metrics}

The most common examples of such metrics are the "uniform convergence" metric $d_{\infty}$ (section 2.1.2) and the Hausdorff metric in its functional version. These two metrics are very similar each other and have properties in common with Asplünd's metric: they generate tolerance tubes and are sensitive to small sized differences.

Nevertheless, their behavior in presence of lighting variations or lighting drift is very different of Asplünd's one. This point is developed in the following section. 
4.1.3. A fundamental advantage: insensitivity to "lighting" variations and "lighting" drift

Let us consider a given template g. For easiness of figures interpretation, we will represent $\mathrm{g}$ as a mono-dimensional function, knowing that the applications we are interested in are bi-dimensional images. On Fig. 13 (a), we can observe homothetic functions $\alpha \otimes g$ of $g$ for various values of $\alpha$, showing that a unique initial template produces a family of probing functions.

Remark: this set of probing functions will permit to adapt the probes to the studied region, giving access to "spatially variant" testing. Another spatially variant approach has been proposed by Bouaynaya et al (Bouaynaya, 2008) in the mathematical morphology framework. This domain may appear disjointed of the probing techniques, but Barat et al have established a strong link between the two contexts in (Barat, 2003) and (Barat, 2010).

The main interest of the novel approach we propose is justified by a physical property of the logarithmic scalar multiplication $\Delta$ : Remember that the LIP Model is initially adapted to images acquired in transmitted light and that $\alpha \otimes g$ represents the image corresponding to an object whose thickness is multiplied by $\alpha$ (Jourlin, 2001) for example.

In such conditions, we can legitimately consider that the set $\{\alpha \otimes g\}$ of probing functions automatically adapts to background illumination variations modelled by "thickness" changing. This point is illustrated (Fig. 13 (a) and 13 (b)). On Fig. 13 (a), we represent a probing function $g$ (in blue color) and two homothetic functions $\alpha \otimes g$ in green and red, respectively with $\alpha>1$ (darker than $g$ ) and $\alpha<1$ (brighter than $g$ ). On 13 (b), the representations of the studied image $f$ and of the probing function $g$ are defined on an interval. On successive intervals $I$, the homothetic functions $\lambda \otimes g$ and $\mu \otimes g$ defining locally the Asplünd's distance between $\left.f\right|_{I}$ and $g$ are drawn. 


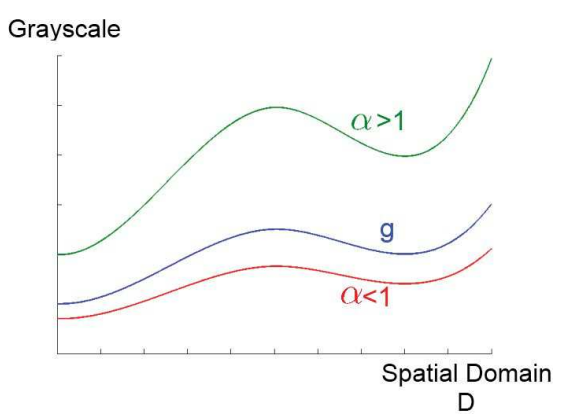

(a)

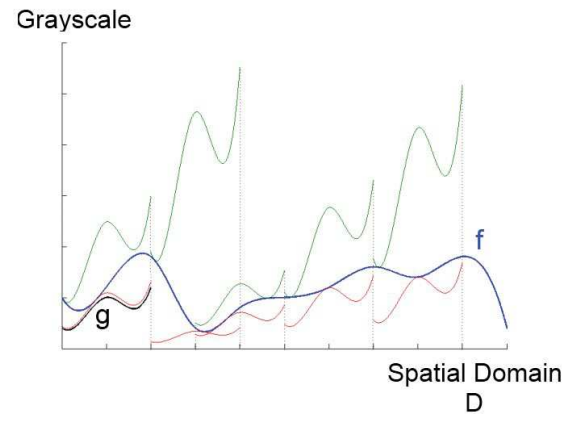

(b)

Figure 13: (a) Probing function g and two homothetic profiles, (b) Profiles of $\lambda \wedge g$ and $\mu \wedge g$ corresponding to $d \frac{\widehat{A}}{A s}\left(\left.f\right|_{I}, g\right)$ minimum

Now let us illustrate this property on a bi-dimensional image $f$, first in the field of images in transmission. For this purpose, we start with the three images presented in Fig. 5 representing dermal papillae acquired in confocal microscopy. The processing used in Fig. 7] (extraction of dermal papillae thanks to Asplünd's metric) is applied exactly in the same conditions to the three images, showing the results are quite similar, despite the strong lighting variations (cf Fig. 14). 


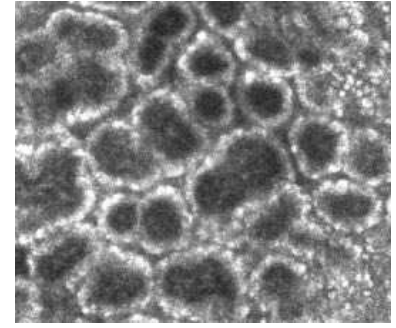

(a)

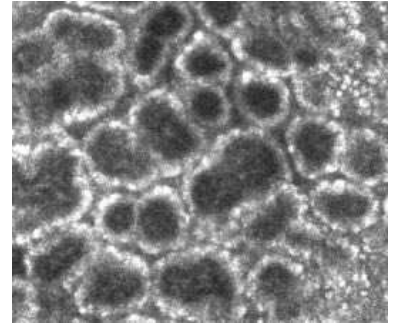

(a)

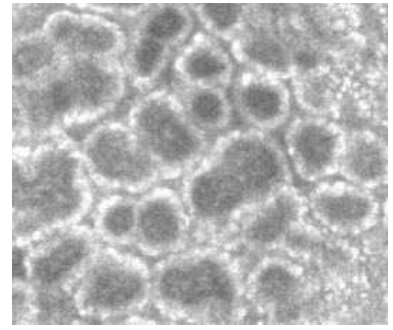

(f)

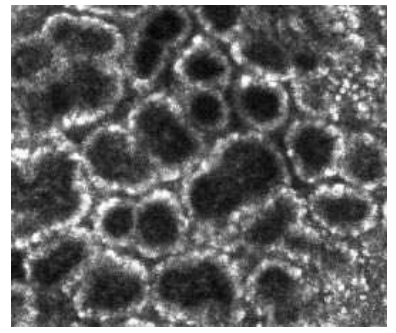

(i)

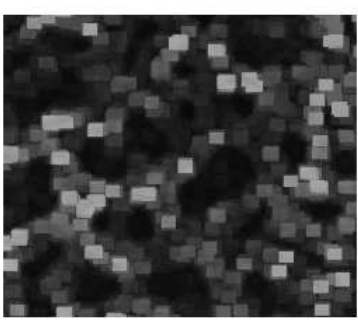

(d)

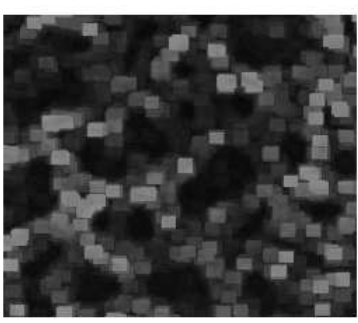

(g)

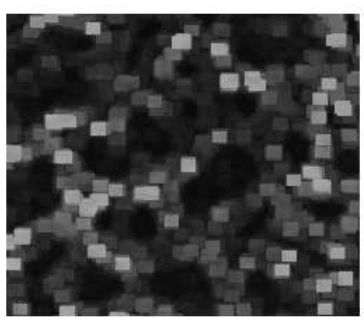

(j) (c)

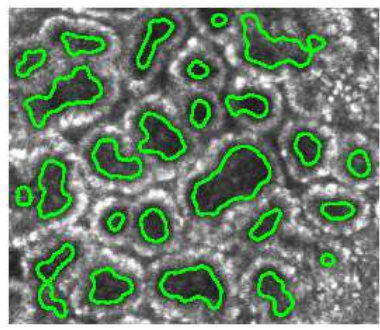

(e)

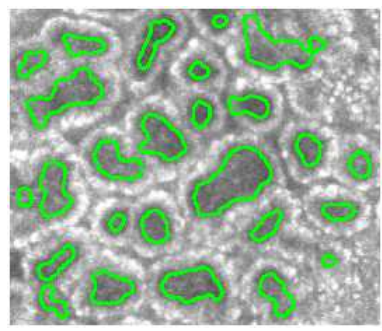

(h)

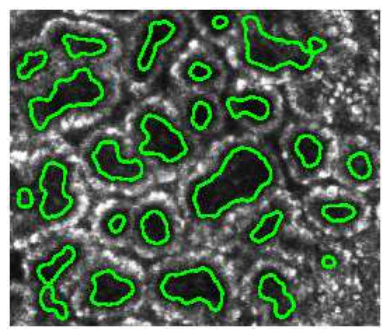

(k)

Figure 14: (a)Initial image of dermal papillae by in-vivo confocal microscopy, (b) Selected target, (c) Magnified target, (d) Asplünd's map for initial image, (e) Contours extracted from (d) and superposed on (a), (f) Brightening of (a), (g) Asplünd's map for brightened image, (h) Contours extracted from (g) and superposed on (f),(i) Darkening of (a), (j) Asplünd's map for darkened image, $(\mathrm{k})$ Contours extracted from $(\mathrm{j})$ and superposed on (i)

Another example is illustrated (cf Fig. 15) for images in reflection. We start with a bricks wall with various gray levels (Fig. 15 (a)). Two bricks with different gray levels are selected as templates $T_{1}$ and $T_{2}$ (Fig. 15 (b) and (e)). 
The resulting Asplünd's maps are given (Fig. 15 (c) and (f)). On these maps, the location of each brick (bright or dark) is clearly pointed by a dark area corresponding to the local minima of Asplünd's metric. Note that such areas would be easily thresholded, due to their strong contrast with their neighboring pixels.

On the same figure, we display the results obtained with the atomic metric $d_{\infty}$ (Fig. 15 (d) and (g)) showing this metric is unable to furnish the location of each brick, particularly for the bright target. 


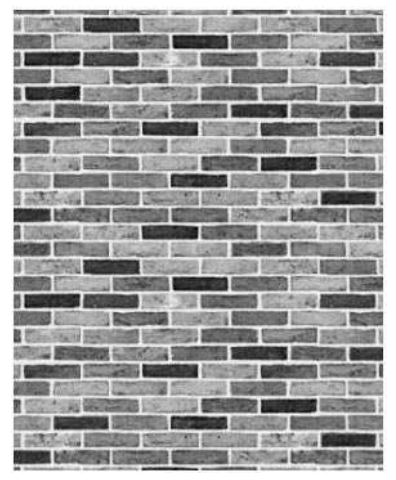

(a)

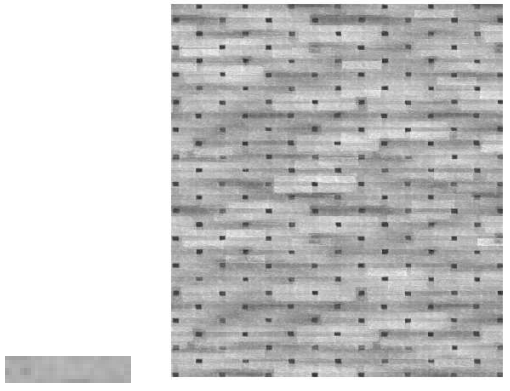

(b)

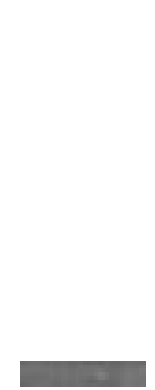

(e) (c)

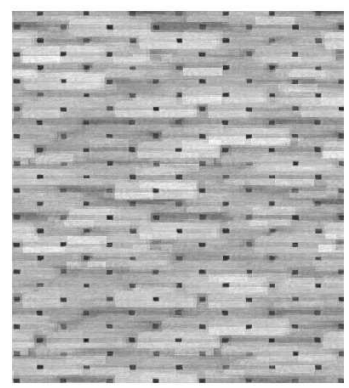

(f)

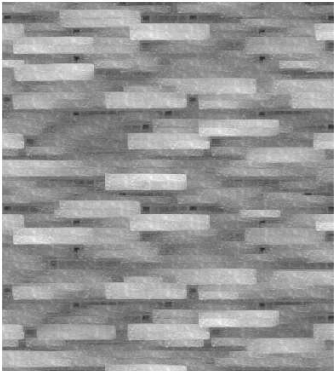

(d)

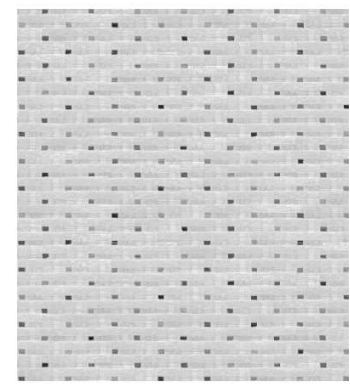

(g)

Figure 15: (a) Initial image, (b) $T_{1}$ magnified two times, (c) Asplünd's map for $T_{1}$, (d) $d_{\infty}$ map for $T_{1}$, (e) $T_{2}$ magnified two times, (f) Asplünd's map for $T_{2}$, (g) $d_{\infty}$ map for $T_{2}$

To conclude this section, let us consider an image presenting a strong lighting drift. We apply to the Petri dish of Escherichia Coli bacteria (Fig. 16 (a)) a lighting drift (Fig. 16 (b)), resulting in Fig. 16 (c). The Asplünd's maps obtained on Fig. 16 (a) and (c) are represented in Fig. 16 (d) and (e), showing the weak sensitivity to lighting drift. 


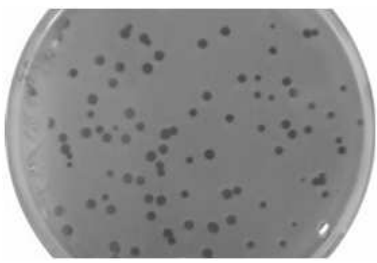

(a)

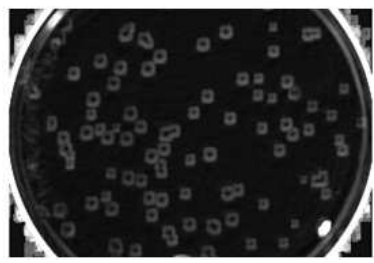

(d)

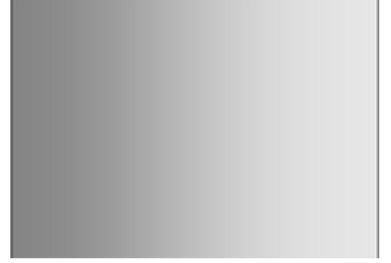

(b)

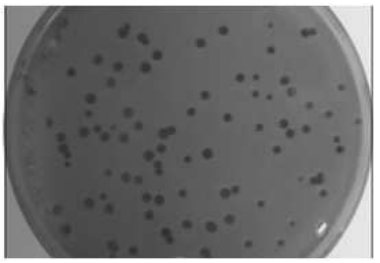

(c)

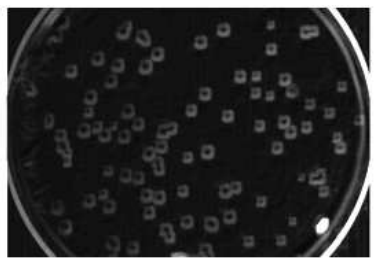

(e)

Figure 16: (a) Petri dish (Escherichia Coli bacteria), (b) Lighting drift, (c) Lighting drift added to (a), (d) Asplünd's map for (a), (e) Asplünd's map for (c)

\subsubsection{Comparison with correlation methods}

Another popular approach to determine the possible locations of a template $T$ inside an image $f$ consists of computing a correlation map (?) thanks to classical correlation coefficient

$$
\frac{\sum_{x \in R}(f(x)-A(R)) \cdot(g(x)-A(T))}{\sqrt{\sum_{x \in R}(f(x)-A(R))^{2}} \cdot \sqrt{\sum_{x \in R}(g(x)-A(T))^{2}}}
$$

where :

- $R$ represents the region of $f$ compared to the target

- $g(y$ the gray level at $y$ in $T$

- $A(R)$ and $A(T)$ the average gray level values of $R$ and $T$

Note that the behavior of this technique is, by definition, similar to that of global metrics, the computation being done on the whole considered data.

We will limit us to show the behavior of a correlation on the Bricks Wall (Fig. 17). 


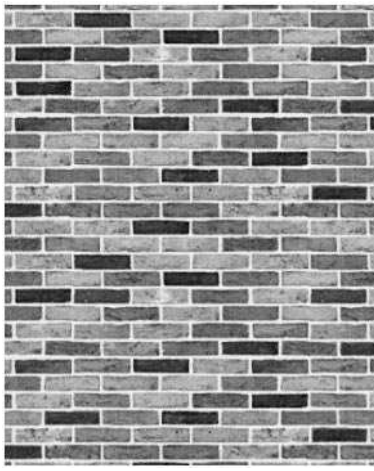

(a)

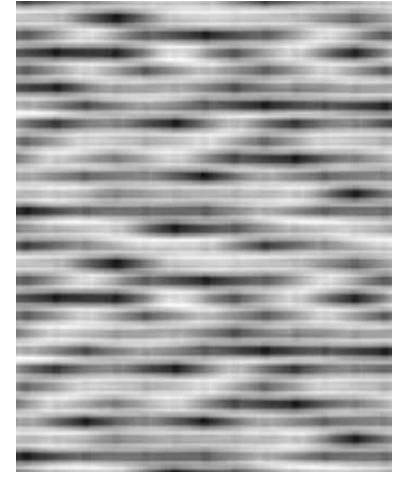

(c)

Figure 17: (a) Initial image, (b) Selected template, (c) Associated correlation map )

Comments on figure 17: this figure clearly shows that a correlation approach will only detect the bright bricks if the target is bright and the dark ones otherwise. Moreover, we can note that the boundaries of the bricks produce weak transitions in terms of gray levels. This point makes difficult the extraction of the peaks on the correlation map.

\subsubsection{Comparison with existing double sided probing}

As previously demonstrated, performing Asplünd's metric clearly appears as a double sided probing, as proposed by Barat (Barat, 2010). The fundamental differences with Barat's approach are mainly due to the physical justification of Asplünd's probes, whose consequences are:

- An automated generation of the probes

- The probes values never come out the gray scale

- The "gap" between probes is not a priori chosen but determined by the probing itself

Excepted these noticeable differences, the behavior and properties of the two methods are similar in the sense they locate a region looking like the template when this region lies inside the tolerance tube determined by the upper and lower templates.

To conclude this section, let us summarize the compared properties of our approach with other metrics and pattern matching techniques in a table (Tab. 11. 


\begin{tabular}{|c|c|c|c|c|c|c|}
\hline \multirow{4}{*}{ Properties } & \multicolumn{6}{|c|}{ Techniques } \\
\hline & \multicolumn{4}{|c|}{ Metrics } & \multirow{3}{*}{$\begin{array}{l}\text { Corre- } \\
\text { lation }\end{array}$} & \multirow{3}{*}{$\begin{array}{l}\text { Double- } \\
\text { sided } \\
\text { probing } \\
\text { (Barat) }\end{array}$} \\
\hline & \multirow{2}{*}{$\begin{array}{l}\text { Global } \\
d_{1}, d_{2}, \\
\ldots, d_{p}\end{array}$} & \multicolumn{3}{|c|}{ Atomic } & & \\
\hline & & $d_{\infty}$ & $d_{H}$ & $d_{A s}^{\wedge}$ & & \\
\hline $\begin{array}{l}\text { Detection of small } \\
\text { sized objects }\end{array}$ & No & Yes & Yes & Yes & No & Yes \\
\hline Tolerance tubes & No & Yes & Yes & Yes & No & Yes \\
\hline Physically justified & No & No & No & Yes & No & No \\
\hline $\begin{array}{l}\text { Insensitivity to } \\
\text { Lighting variations }\end{array}$ & No & No & No & Yes & No & Yes \\
\hline $\begin{array}{ll}\text { Insensitivity } & \text { to } \\
\text { lighting drift } & \end{array}$ & No & No & No & Yes & No & Yes \\
\hline $\begin{array}{l}\text { Insensitivity } \quad \text { to } \\
\text { noise }\end{array}$ & Yes & No & No & No & Yes & No \\
\hline $\begin{array}{l}\text { Automated genera- } \\
\text { tion of probes }\end{array}$ & Not & Conce & & Yes & $\begin{array}{l}\text { Not } \\
\text { Con- } \\
\text { cerned }\end{array}$ & No \\
\hline
\end{tabular}

Table 1: Properties of Asplünd's metric compared to other metrics, to correlation and double sided probing (Barat, 2010)

\subsection{Main drawback: noise sensitivity}

Scientific honesty obligates us to mention that Asplünd's approach is highly sensitive to noise, as well as all other atomic metrics. Nevertheless, an adaptation to image processing of a metric often used in "Measure Theory" will permit us to solve efficiently this problem (see section 5 below).

In order to illustrate the noise sensitivity of the Asplünd's metric, let us consider a gray level image $f$ whose representative surface is an oblique plane (Fig. 18)
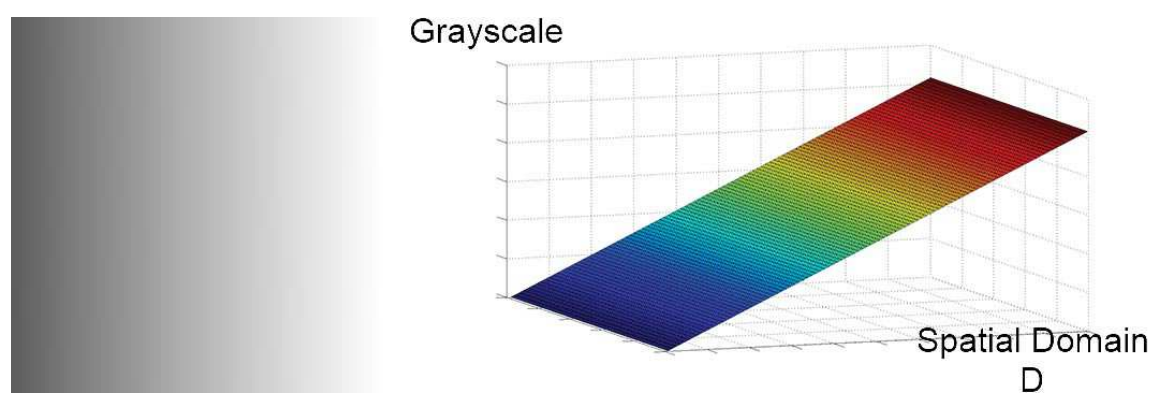

Figure 18: Initial image $f$ and its representative surface in false colors 
On Fig. 19, we can observe the image $g$ corresponding to the previous image $f$ and its representative surface (in false colors) after addition of a Salt and Pepper noise.
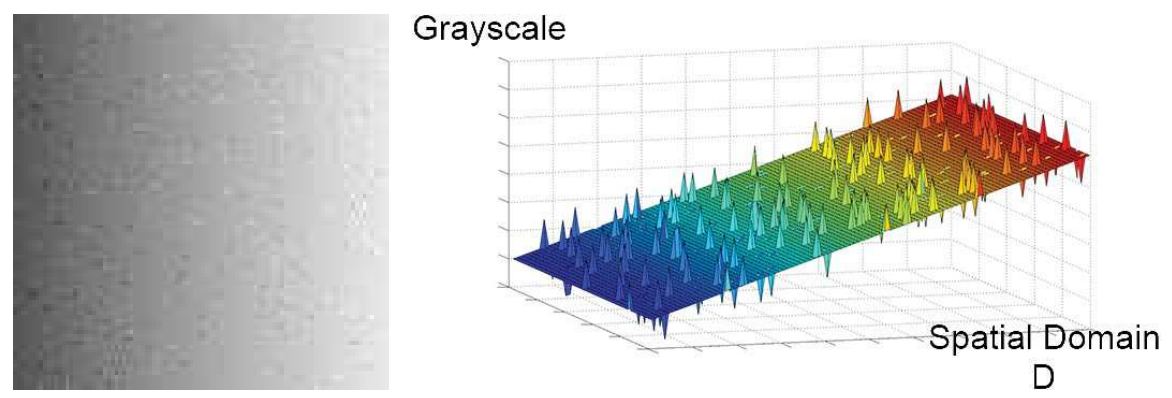

Figure 19: Image $g$ : Initial image $f$ with salt and pepper noise and its representative surface

If we aim at expressing the Asplünd's distance between $f$ and $g$, we compute the real numbers $\lambda$ and $\mu$ corresponding to the probing of $g$ by $f$. The probing oblique planes are represented on both sides of $g$ (Fig. 20). To facilitate interpretation, we limit the representation of Fig. 20 to a section of the representative surfaces.

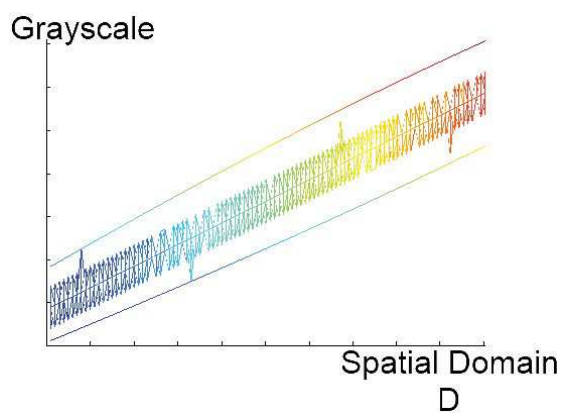

Figure 20: Surfaces of $g$ and the probing functions $\lambda \widehat{x} f$ and $\mu \widehat{x} f$

Noise peaks which determine the Asplünd's distance clearly appear on Fig 20.

\section{A solution to overcome noise sensitivity}

\subsection{Description of the method}

The sensitivity of Asplünd's metric to noise was already mentioned. A very simple way to overcome such a drawback consists of adapting a well-known 
metric to our particular situation. Such a metric has been defined in the context of "Measure Theory" and will be called Measure metric or $M$-metric. Our aim is not here to deeply enter this theory, so we will limit to a short recall adapted to the context of gray level images defined on a subset $D$ of $\mathbb{R}^{2}$. Given a measure $\mu$ on $\mathbb{R}^{2}$, a gray level image $f$ and a metric $\mathrm{d}$ on the space of gray level images, a neighborhood $N_{\mu, d, \epsilon, \epsilon^{\prime}}$ of $f$ may be defined thanks to $\mu$ and to two arbitrary small positive real numbers $\epsilon$ and $\epsilon^{\prime}$ according to:

$$
N_{\mu, d, \epsilon, \epsilon^{\prime}}=\left\{g, \mu(\{x \in D, d(f(x), g(x))>\epsilon\})<\epsilon^{\prime}\right\}
$$

It means that the measure of the set of points $\mathrm{x}$ where $d(f(x), g(x))$ overcomes the tolerance $\epsilon$ satisfies another tolerance $\epsilon^{\prime}$. Let us interpret that in the context of Asplünd's metric:

- the gray level image being digitized, the number of pixels lying in $\mathrm{D}$ is finite, so the "measure" of a subset of $D$ is directly linked to the cardinal of this subset, for example the percentage $P$ of its elements related to $D$ (or a region of interest $R$ included in $D$ ). In our case, we search a subset $D^{\prime}$ of $D$ such that $\left.f\right|_{D^{\prime}}$ and $\left.g\right|_{D^{\prime}}$ are neighbors for the Asplünd's metric and the complementary set $D \backslash D^{\prime}$ of $D^{\prime}$ related to $D$ is small sized when compared to $D$. This last condition can be written:

$$
P\left(D \backslash D^{\prime}\right)=\frac{\#\left(D \backslash D^{\prime}\right)}{\# D} \leq p
$$

where $\mathrm{p}$ represents an acceptable percentage and \#D the number of elements in $D$.

- In such conditions, the neighborhood $N_{\mu, d, \epsilon, \epsilon^{\prime}}(f)$ becomes $N_{P, d_{A s}, \epsilon, p}\left(f^{\prime}\right)$ :

$$
\begin{gathered}
N_{P, d_{A s}, \epsilon, p}\left(f^{\prime}\right)= \\
\left\{g / \exists D^{\prime} \subset D, d_{A s}\left(\left.f\right|_{D^{\prime}},\left.g\right|_{D^{\prime}}\right)<\epsilon \text { and } \frac{\#\left(D \backslash D^{\prime}\right)}{\# D} \leq p\right\}
\end{gathered}
$$

Now let us describe the role of the M-metric concerning the example of the oblique plane (See section 4.2 .

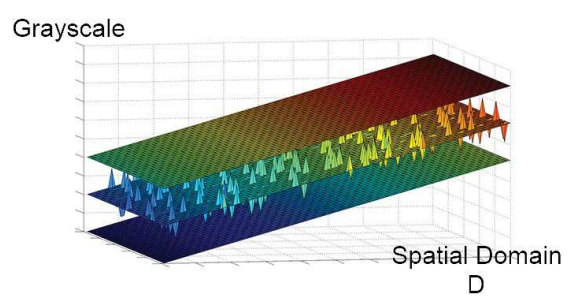

Figure 21: Representative surfaces of $g$ and the probing functions $\lambda \widehat{x} f$ and $\mu \widehat{X} f$ 
In order to decrease the Asplünd's measurement, we need to move closer together the probing functions $\lambda \otimes f$ and $\mu \otimes f$ thanks to the M-metric. Relatively to the definition of this metric, the set $D \backslash D^{\prime}$ corresponds to the part of $D$ where the highest noise peaks are located. Before presenting the details of the method, let us visualize (Fig. 22 (a) and 22 (b)) the set $D \backslash D^{\prime}$ emerging through the probing functions for $p=0.98$ and $p=0.95$.

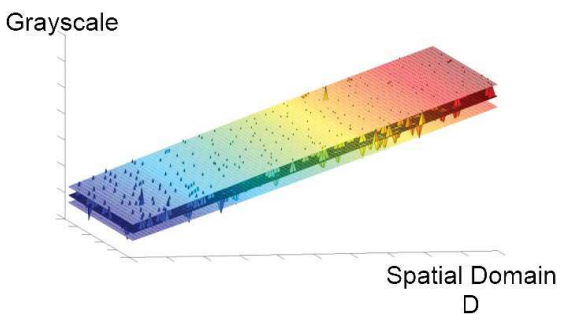

(a)

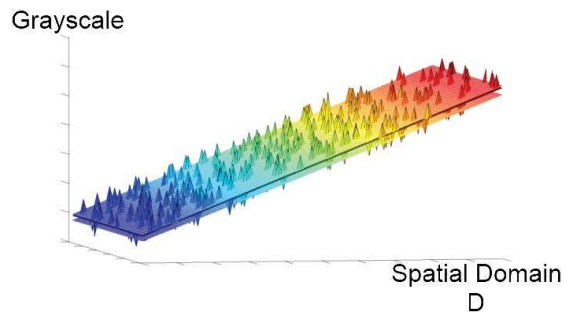

(b)

Figure 22: Decreasing Asplünd's distance by neglecting a small percentage of pixels : (a) Image $g$ and its probing functions for $p=0.98$, (b) Image $g$ and its probing functions for $p=0.95$

The set $D \backslash D^{\prime}$ represents respectively $2 \%$ of the set $D$ (Fig. 22 (a)) and $5 \%$ to the set $D$ (Fig. 22 (b)). It appears that a small restriction of the set $D$ permits to improve highly the Asplünd's distance, and thus to overcome the noise effect.

Associated to the Asplünd's distance, the M-metric permits to determine a set $D^{\prime}$ satisfying the distance (percentage) condition.

Our method uses mainly the differences, pixel per pixel, between two images, and more precisely the histogram of these differences. In our case, this subtraction does not imply any problem: the superior probing function $\lambda \otimes f$ is always superior to the image $g$, which is superior to the inferior probing function $\mu \otimes f$. Thus, the resulting differences will always remain in the grayscale. These histograms are presented in Fig. 23 


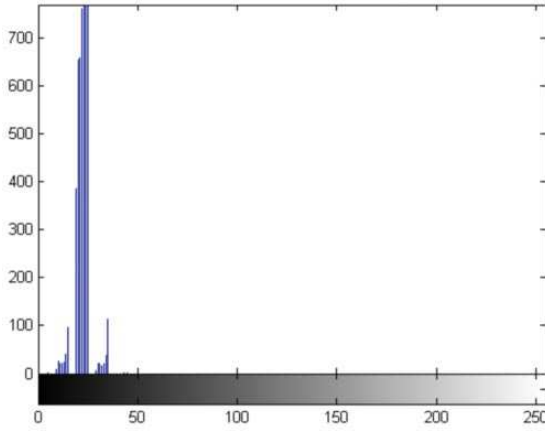

(a)

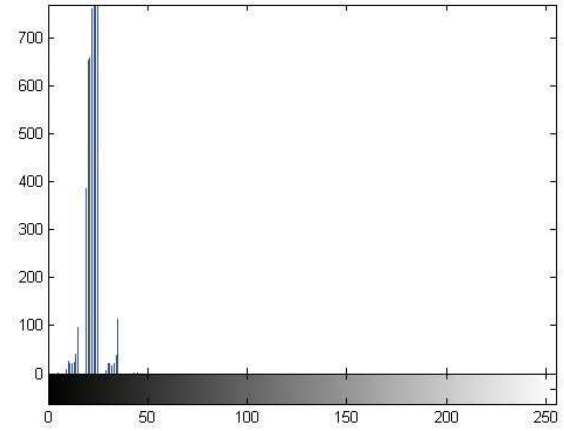

(b)

Figure 23: Histograms of the differences between $g$ and its probing functions : (a) Histogram between $\lambda \widehat{x}_{f}$ and $g$, (b) Histogram between $g$ and $\mu \widehat{x}_{f}$

Note that the first bin of the histogram, corresponding to the value 0 , represents the number of contact pixels between the two images. These histograms reveal that the main part of the pixels between these images present a difference of around 20 gray-levels. As probing functions, $\lambda \otimes f$ and $\mu \otimes f$ must be as close as possible from $g$ in order to decrease the Asplünd's distance, we have to disregard the pixels corresponding to the first bin of the actual histogram of differences, until the bins the most relevant have been reached. Disregard the first bin means to consider the following bin as the new first one. Suppressing the first bin will modify the selection of the pixels making contacts. They will be closer to the image $g$.

Now let us define the real numbers $k_{\lambda}$ and $k_{\mu}$ corresponding to the bins making contact between the images $g$ and $\lambda \otimes f$ and $g$ and $\mu \otimes f$ respectively. Initially, $k_{\lambda}=k_{\mu}=0$ and they are pointing on the real first bin of the histograms. In this configuration, $\lambda$ and $\mu$ correspond to the initial Asplünd's distance. If now we increase $k_{\lambda}$ for instance, a new $\lambda^{\prime}$ can be computed such that the $k_{\lambda}^{\text {th }}$ bin becomes the bin making a contact. In other words, if $x_{\lambda}$ is a pixel belonging to the first bin of the histogram of differences between $g$ and $\lambda \hat{x} f$, we can write:

$$
\lambda \otimes f\left(x_{\lambda}\right)=g\left(x_{\lambda}\right) \text { and } \lambda^{\prime} \otimes f\left(x_{\lambda}\right)=g\left(x_{\lambda}\right)-k_{\lambda}
$$

Similarly, in the case of $k_{\mu}$, if $x_{\mu}$ belongs to the first bin of the histogram of differences between $g$ and $\mu \widehat{\Delta} f$ :

$$
\mu \otimes f\left(x_{\mu}\right)=g\left(x_{\mu}\right) \text { and } \mu^{\prime} \otimes f\left(x_{\mu}\right)=g\left(x_{\mu}\right)+k_{\mu}
$$

The scalars $k_{\lambda}$ and $k_{\mu}$ can be seen as the gray-levels numbers we need to add or subtract in order to get the contact points closer from $g$, as illustrated in Fig. 24. Until the value $\mathrm{p}$ has not been reached, the scalars $k_{\lambda}$ and $k_{\mu}$ are increased. The set $D \backslash D^{\prime}$ corresponds to the pixels belonging to the bins of the histograms 
which are neglected. A new Asplünd's distance is computed for each new pair $\left(\lambda^{\prime}, \mu^{\prime}\right)$.

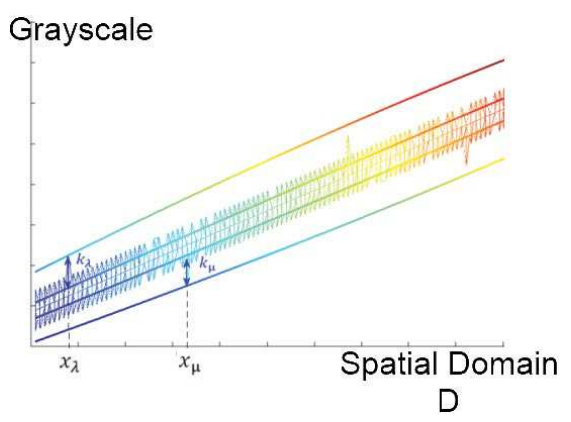

Figure 24: Illustration of the differences $k_{\lambda}$ and $k_{\mu}$ between the initial probing functions and the new ones for $p=0.97$

\subsection{Results}

In Fig. 25, we consider the image "Bricks wall", the same with a Salt and Pepper noise, and the results obtained thanks to the M-metric for various values of the percentage $p$.

Comments on figure 25: These resulting images use the same gray scale. A dark area corresponds to a small value of Asplünd's distance, and the lighter it is, the larger the distance. The resulting images are a little smaller than the initial ones, due to the side effect of the M-metric.

Without the M-metric processing, no information can be measured on the noisy image Fig. 25 (e)). The application of this metric permits to improve the results, and to finally get a quality (Fig. 25 (i), corresponding to the percentage 0.95) comparable to that obtained without any noise.

However, when decreasing the percentage value, we can observe the emergence of horizontal black lines (Fig. 25 (j) and 25(k)). Such lines are due to the fact that the vertical boarders between two successive bricks are small enough to be neglected at the considered percentage.

In conclusion, the M-metric appears efficient to overcome the noise problem. Nevertheless, it remains to find an automated method in order to determine the optimal percentage adapted to a given noisy image. This would probably necessitate an hypothesis on the noise nature.

Another way to approach this problem would consist of studying the curve representing the Asplünd's metric decreasing according to the percentage of neglected points. 


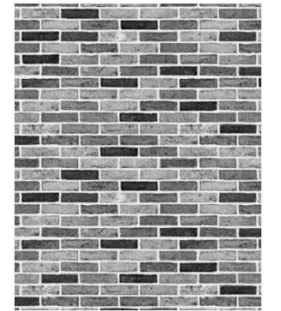

(a)

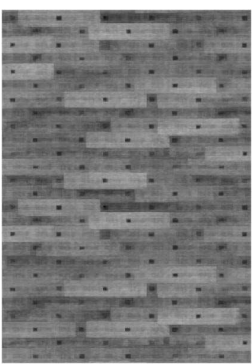

(d) $p=1$

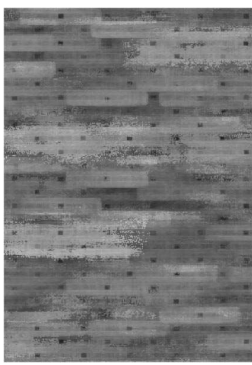

(g) $p=0.98$

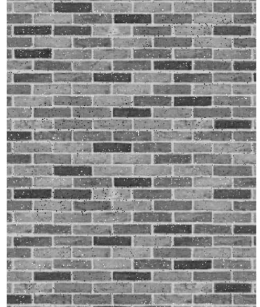

(b)

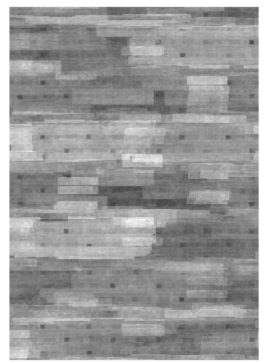

(e) $p=1$

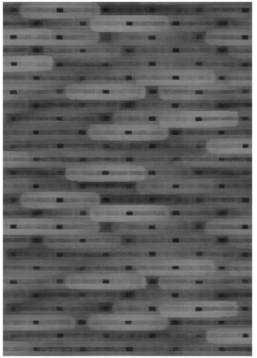

(h) $p=0.95$
(C)

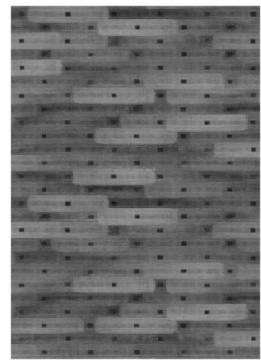

(f) $p=0.98$

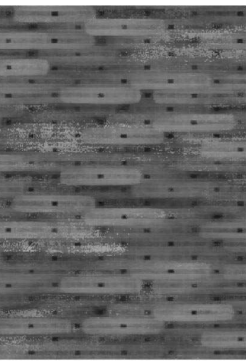

(i) $p=0.95$

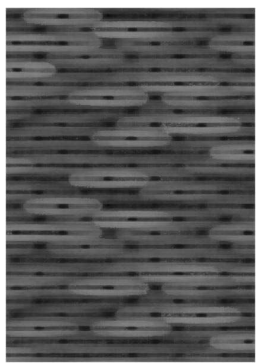

(j) $p=0.90$

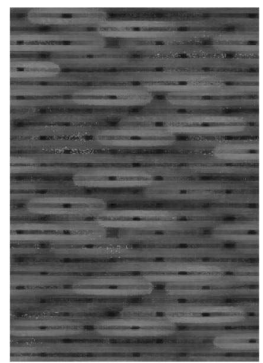

(k) $p=0.90$

Figure 25: Illustration of the M-metric method for various values of percentage p. : (a) Initial image: Brick wall, (b) Initial image with Salt and Pepper noise, (c) Pattern to be extracted (magnified), (d) (f) (h) (j) different values of p for image (a), (e) (g) (i) (k) different values of $\mathrm{p}$ for image (b) 


\section{Conclusion and perspectives}

\subsection{Conclusion}

In the present paper, the little-known Asplünd's metric has been extended to gray level functions in order to detect, inside a studied image, the most probable locations of a given template. The proposed approach appears as a good synthesis between "double sided probing" techniques and "atomic" metrics which present a very strong justification in the field of images acquired in transmitted light.

Moreover, the consistency of the chosen framework (Logarithmic Image Processing Model) with human visual perception permits the application of the method to images acquired in reflection on which we aim at extracting information as a human eye would do.

Asplünd's metric possesses noticeable properties: starting from an initial pattern, the probes are automatically generated and always remain inside the gray scale. Finally, Asplünd's approach has demonstrated its particular efficiency in presence of lighting variations or lighting drift.

The common weakness of Asplünd's metric with probing techniques and atomic metrics resides in their high sensitivity to noise. This problem has been overcome by neglecting a certain percentage of pixels when performing the probing.

\subsection{Perspectives}

The immediate perspective of the present work consists of defining a color version of Asplünd's metric (already done) and applying it to various situations (in progress).

Another natural extension will be to study the links of Asplünd's probing with mathematical morphology, the lower and upper probes allowing clearly the definition of an erosion-type and a dilation-type operators, the 3-dimensional structuring elements corresponding to the representative surfaces of the probes. In order to not disturb the presentation, this point has not been developed here, but its interest is evident, as well as the link with Gauges Theory holding in the Vector Spaces domain (Narici, 1985).

\section{Acknowledgements}

Many thanks are due to the reviewers whose constructive remarks and comments incited us to improve the paper's presentation by introducing in section 4. novel examples and novel references. 


\section{The Bibliography}

\section{References}

E. Asplund, "Comparison between plane symmetric convex bodies and parallelograms," Mathematica Scandinavica, vol. 8, pp. 171-180, 1960.

C. Barat, C. Ducottet, and M. jourlin, "Pattern matching using morphological probing," in Image Processing, 2003. ICIP 2003. Proceedings. 2003 International Conference on, vol. 1, sept. 2003, pp. I - 369-72 vol.1.

C. Barat, C. Ducottet, and M. Jourlin, "Virtual double-sided image probing: A unifying framework for non-linear grayscale pattern matching," Pattern Recogn., vol. 43, no. 10, pp. 3433-3447, Oct. 2010.

N. Bouaynaya and D. Schonfeld, "Theoretical foundations of spatially-variant mathematical morphology part ii: Gray-level images," Pattern Analysis and Machine Intelligence, IEEE Transactions on, vol. 30, no. 5, pp. 837 -850, may 2008.

T. Bozkaya, and M.Ozsoyoglu, "Distance-based indexing for high-dimensional metric spaces", Proceedings of the 1997 ACM SIGMOD international conference on Management of data, pp. 357-368, 1997.

J. Brailean, B. Sullivan, C. Chen, and M. Giger, "Evaluating the em algorithm for image processing using a human visual fidelity criterion," in Proceedings of the International Conference on Acoustics. Speech and Signal Processing, 1991, pp. 2957-2960.

N. Friel and I. S. Molchanov, "Distances between grey-scale images." in Proceedings of the Fourth International Symposium on Mathematical Morphology and Its Applications to Image and Signal Processing Kluwer Academic Publishers, 1998, pp. 283 - 290.

N. Girard, J.M. Ogier and E. Baudrier, "A New Image Quality Measure Considering Perceptual Information and Local Spatial Feature" in Graphics Recognition. Achievements, Challenges, and Evolution, , Ogier, Jean-Marc and Liu, Wenyin and Llados, Josep, Ed. Springer Berlin Heidelberg, 2010, pp. $242-250$.

B. Grünbaum, "Measures of symmetry for convex sets," in Proceedings of Symposia in Pure Mathematics, vol. 7, no. 233-270, 1963.

A. W. Gruen, "Adaptive least squares correlation: A powerful image matching technique", Journal of Photogrammetry, Remote Sensing and Cartography, 14(3),pp. 175187, 1985.

Y. Hel-Or, and H. Hel-Or, "Real-time pattern matching using projection kernels", Pattern Analysis and Machine Intelligence, 27(9), pp. 1430-1445, 2005.. 
D. Huttenlocher, G. Klanderman, and W. Rucklidge, "Comparing images using the hausdorff distance," Pattern Analysis and Machine Intelligence, IEEE Transactions on, vol. 15, no. 9, pp. 850 -863, sep 1993.

M. Jourlin and J.-C. Pinoli, "A model for logarithmic image processing," Journal of Microscopy, vol. 149, no. 1, pp. 21-35, 1988.

M. Jourlin and J. Pinoli, "Logarithmic image processing: The mathematical and physical framework for the representation and processing of transmitted images," in Advances in Imaging and Electron Physics, P. W. Hawkes, Ed. Elsevier, 2001, vol. 115, pp. 129 - 196.

M. Jourlin, M. Carre, J. Breugnot, and M. Bouabdellah, "Chapter 7 - logarithmic image processing: Additive contrast, multiplicative contrast, and associated metrics," in Advances in Imaging and Electron Physics, P. W. Hawkes, Ed. Elsevier, 2012, vol. 171, pp. $357-406$.

T.J. Keating, P.R. Wolf, F.L. Scarpace, "An Improved Method of Digital Image Correlation", in Photogrammetric Engineering and Remote Sensing, Vol. 41, Num. 8, 1975,pp. 993-1002.

D. Lefebvre, H. Arsenault, P. Garcia-Martinez, and C. Ferreira, "Recognition of Unsegmented Targets Invariant under Transformations of Intensity", Applied Optics, 41(29), pp. 6135-6142, 2002.

F. Odone, E. Trucco and A. Verri, "General Purpose Matching of Grey Level Arbitrary Images" in Proceedings of the 4th International Workshop on Visual Form, Springer-Verlag, 2001, pp. 573-582.

N. Otsu, "A threshold selection method from grey-level histograms", in IEEE Transactions on Systems, Man, and Cybernetics, 1979, Vol. 9, Num. 1, pp. $62-66$.

L. Narici,E. Beckenstein, "Topological vector spaces", Vol. 95, 1985), pp. 100124. CRC Press. 